Technological University Dublin ARROW@TU Dublin

\title{
Effects of carbohydrate mouth rinse and caffeine on high- intensity interval running in a fed state
}

\author{
Simon Devenney \\ Technological University Dublin, simon.devenney@tudublin.ie \\ Shane Mangan \\ Technological University Dublin, shane.mangan@tudublin.ie \\ Marcus Shortall \\ Technological University Dublin, marcus.shortall@tudublin.ie
}

See next page for additional authors

Follow this and additional works at: https://arrow.tudublin.ie/ittsciart

Part of the Sports Sciences Commons

\section{Recommended Citation}

Devenney, Simon \& Mangan, Shane \& Collins, Kieran \& Shortall, Marcus. (2017). Effects of carbohydrate mouth rinse and caffeine on high intensity interval running in a fed state. Applied Physiology Nutrition and Metabolism. 43. 10.1139/apnm-2017-0458.

This Article is brought to you for free and open access by the School of Science and Computing at ARROW@TU Dublin. It has been accepted for inclusion in Articles by an authorized administrator of ARROW@TU Dublin. For more information, please contact arrow.admin@tudublin.ie, aisling.coyne@tudublin.ie, gerard.connolly@tudublin.ie.

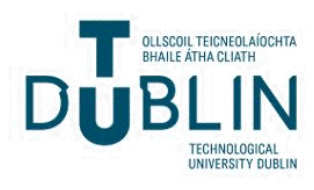




\section{Authors}

Simon Devenney, Shane Mangan, Marcus Shortall, and Kieran Collins

This article is available at ARROW@TU Dublin: https://arrow.tudublin.ie/ittsciart/106 


\title{
Effects of carbohydrate mouth rinse and caffeine on high-intensity interval running in a fed state
}

\author{
Simon Devenney, Shane Mangan, Marcus Shortall, and Kieran Collins
}

\begin{abstract}
The current study aims to identify if mouth rinsing with a $6 \%$ carbohydrate mouth-rinse (CMR) solution and mouth rinsing and ingestion of caffeine (CMR+CAFF) can affect exercise performance during steady-state (SS) running and highintensity intervals (HIIT) in comparison with a $0 \%$ control solution (PLA) when in a fed state. Eight recreationally trained males completed 3 trials (CMR, CMR+CAFF, and PLA) of 45 min SS running and an HIT protocol ( $90 \%$ peak treadmill velocity) until fatigue in a double blinded, repeated-measures study. Participants ingested a capsule of either CAFF or PLA before and after SS. Participants received a 25-mL bolus of carbohydrate solution (CMR and CMR+CAFF trials) or taste-matched PLA (PLA trial) prior to HIIT protocol and after every second effort. Heart rate and lactate responses were recorded throughout the SS and HIIT protocol. CMR+CAFF was significantly different when compared with PLA $(p=0.001$; Cohens $d=1.34)$ and CMR $(p=0.031$; Cohens $d=0.87$ ) in relation to distance covered before fatigue. Although there was no significant difference between CMR and PLA, there was a small benefit for CMR $(p=0.218$; Cohens $d=0.46$ ). Results indicate that CMR and ingestion of CAFF leads to improvements in performance during interval sessions while participants were in a fed state. These findings indicate that the regular use of CMR can decrease the risk of gastrointestinal distress reported by athletes, which can be applicable to athletes in a real-world setting.
\end{abstract}

Key words: mouth rinse, carbohydrate, caffeine, high intensity, interval running.

Résumé : Dans cette étude, on vérifie si l'utilisation d'un rince-bouche contenant $6 \%$ de sucre ("CMR ») et l'utilisation d'un rince-bouche combinée à la consommation de caféine ("CMR+CAFF ») ont un effet sur la performance au cours d'une séance de course en régime stable (SS) et par intervalles d'intensité élevée ( HIIT ») comparativement à une solution de contrôle contenant $0 \%$ d'ingrédient ("PLA ») sans être à jeun. Huit hommes actifs par loisir participent, selon un devis à double insu avec mesures répétées, à trois essais (CMR, CMR+CAFF et PLA) comportant 45 min de course SS suivie d'un HIIT (90\% de la vitesse de pointe sur tapis roulant) jusqu'à épuisement. Les participants consomment une capsule du placebo ou de caféine avant et après SS. Les participants consomment $25 \mathrm{~mL}$ de solution sucrée (essais CMR et CMR+CAFF) ou d'un placebo ayant le même goût (essai PLA) avant le protocole HIIT et après chaque deuxième effort. On enregistre les valeurs de la fréquence cardiaque et de la concentration de lactate tout au long de SS et du protocole HIIT. La condition CMR+CAFF révèle des résultats significativement différents comparativement aux conditions PLA ( $p=0,001 ; d$ de Cohen $=1,34)$ et CMR $(p=0,031 ; d$ de Cohen $=0,87)$ en ce qui concerne la distance franchie avant l'épuisement. Bien qu'il n'y ait pas de différence significative entre CMR et PLA, la condition CMR présente un léger bénéfice $(p=0,218$; $d$ de Cohen $=0,46)$. D'après les résultats, la condition CMR+CAFF suscite une amélioration de la performance au cours des intervalles de course chez les participants non à jeun. D'après les observations, l'utilisation régulière d'un CMR peut diminuer le risque de malaises gastro-intestinaux rapportés par les athlètes; ces observations pourraient s'appliquer aux athlètes en milieu naturel. [Traduit par la Rédaction]

Mots-clés : rince-bouche, sucre, caféine, intensité élevée, course par intervalle.

\section{Introduction}

Early carbohydrate mouth-rinse (CMR) studies reported improvements in both running and cycling time trial performance (Carter et al. 2004; Pottier et al. 2010; Rollo and Williams 2011; Rollo et al. 2008). Carter et al. (2004) investigated the effects of a $6.4 \%$ maltodextrin solution on a 1-h time trial in comparison with a $0 \%$ solution. Improvements of $2.9 \%$ were reported for the average time to completion and the authors hypothesised that CMR's improvements in time-trial performance is linked to the activation of the neural pathways via the oral receptors (Chambers et al. 2009).

Whilst CMR studies have primarily been focused on cycling performance (Beelen et al. 2009; Carter et al. 2004; Chong et al.
2011; Lesniak et al. 2016), studies have shifted some focus to investigating the effects of CMR on other endurance sports (Dorling and Earnest 2013; Kasper et al. 2016; Rollo et al. 2008, 2010). Following an overnight fast, Rollo et al. (2008) investigated the effects of CMR on running performance with results showing a positive effect in relation to running speeds and total distance covered due to the CMR. Not all the findings from studies investigating CMR have been positive, as work by Beelen et al. (2009) showed no enhancement in power output, heart rate, or time to completion with a CMR (6.4\% maltodextrin) whilst individuals were in a fed state. More recent work by Devenney et al (2016) demonstrated that when in a fed state, a 6\% CMR (maltodextrin) can elicit a 5.6\% performance improvement in cycling performance. With the effects of $6 \%$ and $6.4 \%$ solutions well documented, more recent stud-

Received 21 July 2017. Accepted 20 November 2017.

S. Devenney, S. Mangan, M. Shortall, and K. Collins. Department of Science, Centre for Exercise and Metabolic Science, Institute of Technology Tallaght, Dublin, Ireland.

Corresponding author: Simon Devenney (email: Simon.Devenney@it-tallaght.ie).

Copyright remains with the author(s) or their institution(s). Permission for reuse (free in most cases) can be obtained from RightsLink. 
ies have investigated higher concentrations CMR (Devenney et al. 2016; Lane et al. 2013). Improvements of $1.8 \%$ were reported in time-trial performance with a $10 \%$ solution in a fed state, while improvements of $3 \%$ were reported in a fasted state (Lane et al. 2013), while Devenney et al. (2016) reported improvements of up to $6.1 \%$ with a $16 \%$ solution when compared with a $0 \%$ placebo (PLA). These findings compare favourably to previous studies of $6 \%$ and $6.4 \%$ solutions, showing increases in exercise performance linked to oral receptor activation (Chambers et al. 2009; Frank et al. 2008). These authors found that the use of CMR increased activation of the reward region of the brain, mainly the ventral striatum and anterior cingulate cortex (Chambers et al. 2009; Frank et al. 2008).

Caffeine (CAFF) ingestion can enhance repeated sprint performance in both running (Carr et al. 2008; Mohr et al. 2011) and cycling (Lee et al. 2011). Kalmar (2005) reported that this endurance and sprint performance enhancement may be due to a mechanism of motor unit activity along with adenosine receptor antagonism. Recent work by Kasper et al. (2016) investigated the effects of CMR and the supplementation of CAFF on anaerobic running capacity in a glycogen depleted state. Participants completed a 45-min steady-state (SS) (65\% maximal oxygen uptake $\left.\left(\dot{V} \mathrm{O}_{2 \mathrm{max}}\right)\right)$ run followed by an HIT exercise capacity $(1 \mathrm{~min}$ at $80 \%$ $\dot{V} \mathrm{O}_{2 \max }$ interspersed by $1 \mathrm{~min}$ at $60 \% \dot{\mathrm{V}} \mathrm{O}_{2 \max }$ ) until fatigue. The findings demonstrated an increased exercise capacity with CMR and CAFF ingestion (CMR+CAFF) when compared with a PLA and CMR by itself. Other work by Kizzi et al. (2016) investigated the effects of a CAFF rinse compared with both a control and PLA while participants were in a low endogenous carbohydrate state. Although the study showed that peak power output was greater in the control, CAFF rinse prevented a drop-off in power outputs across a repeated-cycling sprint protocol in comparison with both the control and PLA. Whilst the majority of the previously mentioned research has been carried out when athletes are in a fasted state, there is a greater need for research findings demonstrating the benefits of CMR in a fed state, as it is rare that athletes will endure competition in a fasted state. Although recent work by Lane et al. (2013) and Devenney et al. (2016) has begun to show the benefits of CMR in a fed state, more detailed work in the area is needed. The aim of this study was to ascertain whether CMR, either alone or in combination with CAFF, could enhance the high-intensity running performance of recreationally trained athletes in a fed state, when compared with a PLA.

\section{Materials and methods}

\section{Recruitment}

Eight recreationally trained males volunteered to participate in this study (age: $23 \pm 3$ years; body mass: $78 \pm 7 \mathrm{~kg}$; height: $1.75 \pm 0.05 \mathrm{~m}$; body mass index: $22 \pm 1.7 \mathrm{~kg} \cdot \mathrm{m}^{-2} ; \dot{V O}_{2 \max }: 51 \pm 3 \mathrm{~mL} \cdot \mathrm{kg}^{-1} \cdot \mathrm{min}^{-1}$ ), all of whom had engaged in exercise of $5-8 \mathrm{~h}$ per week. All participants were informed of the study both verbally and in written form along with an outline of the physiological demands for each testing session. Medical questionnaire and consent forms were completed for each participant prior to testing. The study was approved by the Ethics Committee of Institute of Technology Tallaght.

\section{Overall study design}

In a randomised, repeated-measures and double-blinded study participants undertook an SS run (45 min at 65\% peak treadmill velocity (PTV)) followed by an HIIT protocol until exhaustion (1-min bouts at $90 \%$ PTV, separated by a 1-min bout of walking at $\left.6 \mathrm{~km} \cdot \mathrm{h}^{-1}\right)$. Participants consumed a standardised CAFF capsule (200 mg) or capsulated PLA prior to the SS running and a second CAFF or PLA capsule was consumed prior to the commencement of the HIIT protocol. Throughout the HIIT protocol participants rinsed with a $6 \%$ carbohydrate (maltodextrin) solution for $5 \mathrm{~s}$ on completion of every second interval. Therefore, each participant
Fig. 1. Schematic overview of the testing session. CMR, carbohydrate mouth rinse; HIIT, high-intensity interval training; PTV, peak treadmill velocity.

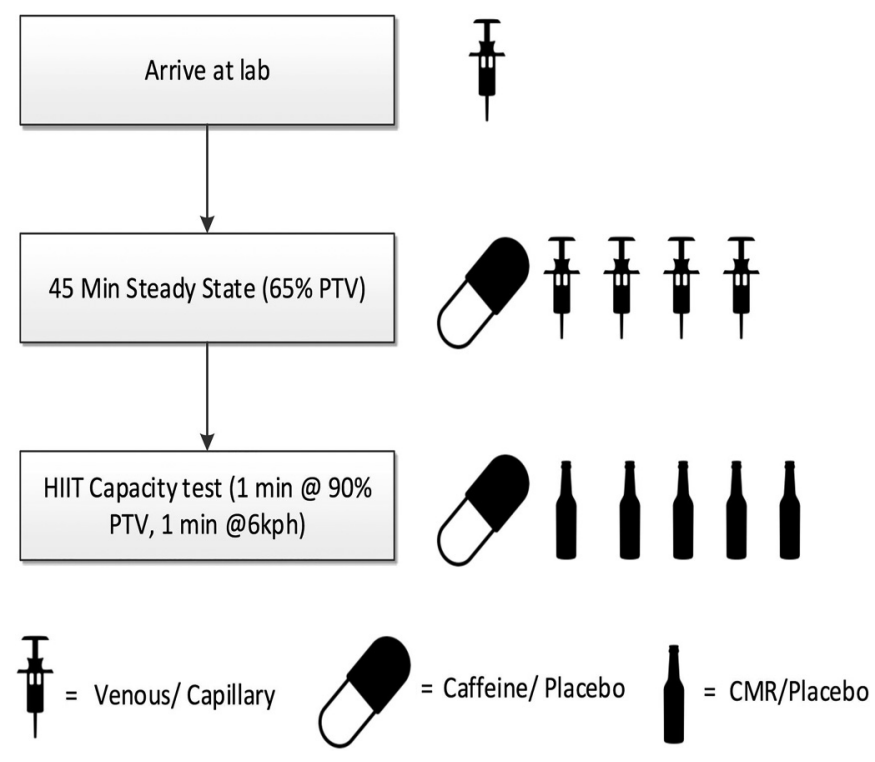

undertook 3 experimental trials that consisted of PLA capsule and PLA mouth rinse (PLA trial), PLA capsule and CMR (CMR trial), and CAFF capsule and CMR (CMR+CAFF trial). The primary marker during this study was time to fatigue in the HIIT protocol. Heart rate (HR), blood lactate, and rate of perceived exertion were monitored throughout the SS run, while HR responses were monitored throughout the HIIT protocol. Figure 1 gives an overview of the experimental design.

\section{Assessment of $\dot{V} \mathrm{O}_{2 \max }$}

A week prior to their first trial, each participant completed a graded exercise test on a motorised treadmill (Cosmed T170, Roma, Italy) to determine $\dot{\mathrm{VO}}_{2 \text { max }}$. The protocol used was based on that of Akubat et al. (2014), with 4-min stages interspersed with 1-min recovery. Stages started at $8 \mathrm{~km} / \mathrm{h}$ with increments of $2 \mathrm{~km} / \mathrm{h}$ after completion of each stage $\left(8,10,12,14,16 \mathrm{~km} \cdot \mathrm{h}^{-1}\right)$ until onset of blood lactate accumulation, which was followed by a ramp protocol (increments of $1 \mathrm{~km} \cdot \mathrm{h}^{-1} \cdot \mathrm{min}^{-1}$ ) to volatile exhaustion. Breath by breath analysis was recorded throughout the test using the Cosmed Quark CPET Metabolic Cart, Gas Analyser (Cosmed, Roma, Italy) with achievement of $\dot{V}_{2 \max }$ determined by plateau of oxygen consumption despite an increase of workload and a respiratory exchange ratio value greater than 1.1. Running speeds for the experimental trials were determined from PTV. Following graded exercise tests, participants undertook a familiarisation process for the speeds at both the SS (65\% PTV) and HIIT (90\% PTV), so participants could familiarize themselves to the demands required in the remaining 3 visits.

\section{SS exercise protocol and HIIT protocol}

Kasper et al. (2016) implemented a 45-min SS and chose to administer CAFF prior to the SS based on findings from Graham and Spriet (1995), which demonstrated that peak plasma CAFF typically occur 45-min after ingestion. Therefore, this study implemented a similar 45-min SS set at 65\% PTV for each participant. Participants arrived at the laboratory at their allocated time and were given either $200 \mathrm{mg}$ of CAFF (Bulk Powders, Essex, UK) or a visually identical PLA (Whey Protein Isolate; Bulk Powders) prior to, and immediately after, the completion of the SS exercise protocol. The second CAFF capsule was administered prior to the HIIT protocol as a means of further developing the ergogenic effect. HR, rating of perceived exertion (RPE) (Borg 1982; Borg et al. 1987), 
and blood lactate were monitored throughout the SS exercise at time points of $5,10,20,30,40$, and $45 \mathrm{~min}$. Following the SS protocol, participants commenced an HIIT protocol that consisted of 1-min bouts at $90 \%$ PTV followed by a 1-min bout of walking at $6 \mathrm{~km} \cdot \mathrm{h}^{-1}$ until physical exhaustion. Time to fatigue was calculated at the time which the participant could no longer meet the demands of the protocol. This follows similar methodology of prescribing interval where specific velocities are used to prescribe intervals (Buchheit and Laursen 2013) Participants rinsed a 25-mL bolus containing either a $6 \%$ carbohydrate beverage (Maltodextrin; Bulk Powders) or a taste-matched (orange) and visually identical PLA (Robinson Squash; Britvic Orange Soft Drinks, Hertsfordshire, UK) for 5 -s periods every 4 min during exercise before expectorating.

\section{Activity and diet before experiments}

A 2-day training diary was kept by each participant prior to each test visit, with low-intensity exercise (HR below 150 beats. min $^{-1}$ ) permitted for up to $60 \mathrm{~min}$ the day prior to testing. Alcohol and CAFF consumption was not permitted in the $24 \mathrm{~h}$ prior to each visit. Dietary intake was recorded and was replicated for each visit as a measure to prevent the disruption of the results. Participants consumed a meal $2-3 \mathrm{~h}$ before testing ( $49 \% \pm 2 \%$ carbohydrates, $18 \% \pm 1 \%$ protein, and $33 \% \pm 2 \%$ fat) with each meal recommended to the participants by the authors.

\section{Statistics}

Data collected were analysed using a 1-way repeated-measures ANOVA (IBM SPSS, version 24 for Windows; IBM Corp.). Before running the required ANOVA to investigate differences in exercise capacity (distance running during HIIT protocol) we performed a Shapiro-Wilks test to assess the data for normality. In the event that any statistically significant differences arose, a post hoc analysis was conducted comprising a paired $t$ test with a Bonferroni adjustment of the level of alpha for multiple comparisons. Values are expressed as the means \pm SD with significance set at $p<0.05$. Effect sizes (ESs) and $95 \%$ confidence intervals (95\% CIs) are reported for applicability to the general population. ESs are reported in line with previous recommendations (Cohen 1992; Lakens 2013), using thresholds of 0.2 (small), 0.6 (moderate), and 1.2 (large) (Hopkins et al. 2009) and effects of 0.19 or less deemed trivial.

\section{Results}

\section{Distance to fatigue}

In relation to running performance there was a significant increase in distance covered between the PLA and the CMR+CAFF ( $p=0.001$, 95\% CI: 1036-2645 m; Cohen's $d=1.34)$ and between CMR and CMR+CAFF ( $p=0.031,95 \%$ CI: 131-2367 m; Cohen's $d=0.87)$. However, there was no significant difference reported between the PLA and CMR ( $p=0.218,95 \%$ CI: -302 to $1484 \mathrm{~m}$; Cohen's $d=0.46$ ), although ES can indicate a possible benefit. Mean distance covered across the individual trials were as follows: PLA, $4535 \pm 1217$ (95\% CI: 3634-5438) m; CMR, $5127 \pm 1367$ (95\% CI: 4114-6140) m; CMR+CAFF, $6376 \pm 1508$ (95\% CI: 5259-7493) $\mathrm{m}$ and are illustrated in Fig. 2.

\section{Physiological responses}

Values for HR were seen to increase steadily with the onset of exercise across the 3 trials with Table 1 illustrating HR responses at set time points. There was no significant difference in heart rate responses across the 3 trials $(p>0.05)$.

Similarly to HR, lactate responses remained constant throughout the SS running, while an increase was seen post-HIIT across all 3 trials. No significance was observed when comparing lactate responses between the experimental trials $(p>0.05)$. Again similarly to HR and blood lactate, no significance was reported in RPE scores across all trials $(p>0.05)$.
Fig. 2. Mean distance to fatigue during the HIIT exercise test across all experimental trials. *, A possible benefit compared with the PLA; $\dagger$, a significant benefit compared with the PLA. CAFF, caffeine ingestion; CMR, carbohydrate mouth rinse; HIIT, high-intensity interval training; PLA, placebo.

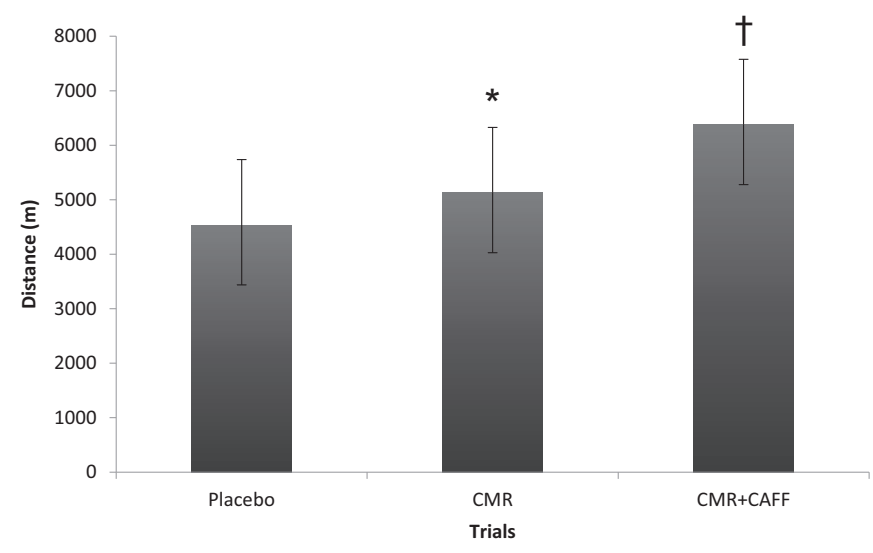

\section{Rinse solution detection}

None of the participants involved in the study were able to differentiate between the mouth rinsing solutions used across the 3 experimental trials. Each participant was unable to distinguish any difference in taste or viscosity of the solutions.

\section{Discussion}

The aim of the study was to ascertain whether CMR, either alone or in addition to CAFF supplementation, could augment high-intensity running performance in comparison with a PLA in recreationally trained males in a fed state. The findings from the study support the initial hypothesis of incremental benefit to running performance, similar to that of previous work by Kasper et al. (2016). Although the statistical certainty around the improvements in the CMR group are not as strong $(p=0.218)$ in relation to distance to fatigue, the CMR does illicit a small to moderate effect (Cohen's $d=0.46$ ). We also confirm that CAFF maintains its ergogenic effects despite exercising in a fed state.

Previous work examining the effect of CMR in a postprandial state has been somewhat equivocal. Early work revealed no significant effect of CMR on exercise performance in a postprandial state (Beelen et al. 2009; Whitham and McKinney 2007) while more recent work has shown performance improvements of $1.8 \%$ 7.1\% (Devenney et al. 2016; Lane et al. 2013) albeit with higher solution concentrations in conjunction to the more traditional CMR concentrations. While the CMR did not produce a statistically significant benefit, the different CIs and moderate Cohen's $d$ ESs would suggest that there is still a benefit elicited from the CMR condition. This would support previous work that has demonstrated that while there seems to be greater benefit in a fasted state, a benefit is still obtained from CMR even in the fed state (Devenney et al. 2016; Fares and Kayser 2011; Lane et al. 2013).

In an bid to further enhance exercise performance, CAFF capsules were consumed both pre- and post-SS sessions to allow peak plasma levels before the commencement of the HIIT protocol (Goldstein et al. 2010). Compared with a PLA, this addition of CAFF had a profound effect on running distance with significant difference in distance to fatigue and a large ES (Cohen's $d=1.34$ ). This would indicate strong merit in the prescription of CMR+CAFF for those undertaking high-intensity running. Furthermore, not only did the CMR+CAFF condition have a significant benefit over the PLA condition, but a significant increase in running distance when compared with the CMR-only condition was also observed. Thus it seems the addition of CAFF exerts a moderate effect (Cohen's $d=0.87$ ) on high-intensity running performance. This 
Table 1. Mean HR, blood lactate, and RPE values across the 3 experimental trials.

\begin{tabular}{|c|c|c|c|c|c|c|c|}
\hline & \multicolumn{6}{|c|}{ Time (min) } & \multirow{2}{*}{$\begin{array}{l}\text { Exhaustion } \\
\text { (min) }\end{array}$} \\
\hline & 5 & 10 & 20 & 30 & 40 & 45 & \\
\hline \multicolumn{8}{|l|}{ Placebo } \\
\hline HR (beats. $\min ^{-1}$ ) & $155 \pm 15$ & $162 \pm 14$ & $167 \pm 17$ & $171 \pm 13$ & $171 \pm 15$ & $172 \pm 15$ & $188 \pm 12$ \\
\hline Lactate $\left(\mathrm{mmol} \cdot \mathrm{L}^{-1}\right)$ & $2.2 \pm 0.9$ & $2.3 \pm 1.0$ & $2.5 \pm 1.1$ & $2.5 \pm 1.2$ & $2.5 \pm 1.1$ & $2.4 \pm 1.2$ & $9.8 \pm 2.3$ \\
\hline RPE (Borg scale) & $10 \pm 2$ & $11 \pm 3$ & $12 \pm 2$ & $14 \pm 3$ & $14 \pm 2$ & $14 \pm 2$ & $20 \pm 1$ \\
\hline \multicolumn{8}{|l|}{ CMR } \\
\hline HR (beats· $\min ^{-1}$ ) & $151 \pm 10$ & $160 \pm 11$ & $164 \pm 10$ & $169 \pm 12$ & $169 \pm 12$ & $170 \pm 13$ & $188 \pm 10$ \\
\hline Lactate $\left(\mathrm{mmol} \cdot \mathrm{L}^{-1}\right)$ & $1.9 \pm 0.6$ & $2.1 \pm 0.9$ & $2.1 \pm 0.9$ & $2.1 \pm 1.1$ & $2.1 \pm 1.1$ & $2.2 \pm 1.1$ & $9.5 \pm 3.0$ \\
\hline RPE (Borg scale) & $9 \pm 2$ & $9 \pm 3$ & $11 \pm 2$ & $13 \pm 2$ & $14 \pm 2$ & $13 \pm 3$ & $20 \pm 1$ \\
\hline \multicolumn{8}{|l|}{ CMR+CAFF } \\
\hline HR (beats. $\min ^{-1}$ ) & $150 \pm 12$ & $160 \pm 17$ & $168 \pm 16$ & $171 \pm 17$ & $173 \pm 16$ & $174 \pm 16$ & $192 \pm 10$ \\
\hline Lactate $\left(\mathrm{mmol} \cdot \mathrm{L}^{-1}\right)$ & $1.9 \pm 0.9$ & $2.0 \pm 0.9$ & $2.4 \pm 1.1$ & $2.5 \pm 1.0$ & $2.6 \pm 1.0$ & $2.3 \pm 1.1$ & $10.1 \pm 2.4$ \\
\hline RPE (Borg scale) & $9 \pm 2$ & $10 \pm 2$ & $11 \pm 1$ & $12 \pm 3$ & $13 \pm 3$ & $13 \pm 2$ & $20 \pm 1$ \\
\hline
\end{tabular}

Note: CAFF, caffeine ingestion; CMR, carbohydrate mouth-rinse; HR, heart rate; RPE, rating of perceived exhaustion.

study, alongside the work of Kasper et al. (2016), provides compelling evidence for the addition of CAFF to CMR to further augment performance. CMR is believed to stimulate the reward pathways of the brain via oral receptors owing to a carbohydrate solutions caloric content (Chambers et al. 2009; Turner et al. 2014). One would envision that this stimulation would illicit lower RPE levels in the CMR trials compared with the PLA trial, leading to a viable reason for the increase in exercise performance, as exercise intensity or tolerance can increase to compensate for the drop in RPE levels. However, previous research has reported a lack of a difference in RPE and HR responses (Beaven et al. 2013; Carter et al. 2004; Chambers et al. 2009; Devenney et al. 2016; Pottier et al. 2010) and the current study confirms the findings, with no significant difference reported in RPE or HR levels between either of the CMR or CMR+CAFF trials and the PLA trial.

There were limitations to consider within the current study. As participants were recreationally trained athletes, it is unknown if the current results can translate to the well-trained athletic population. An additional limitation of this study is the sample size $(n=8)$; therefore, the authors utilised ES to understand the practical significance of the results. Although practical significance was found, the authors issue caution owing to the small sample size. Although subjects were reported to be low consumers of CAFF, CAFF habituation was not measured and therefore may have affected acute responses to the CAFF dose. There was no utilization of a non-rinse control, nor was the possibility of a dose-response addressed.

\section{Conclusion}

In conclusion, the study provides novel data that illustrates that CMR, whether alone or in tangent with caffeine supplementation, can delay the onset of fatigue in anaerobic interval running. With the benefits already reported, these findings along with previous research suggest that the regular use of CMR can decrease the risk of gastrointestinal distress reported by athletes, meaning the data can be applicable to both athletes and coaches in a real-world setting.

\section{Conflict of interest statement}

The authors report no conflicts of interest associated with this manuscript.

\section{References}

Akubat, I., Barrett, S., and Abt, G. 2014. Integrating the internal and external training loads in soccer. Int. J. Sports Physiol. Perform. 9(3): 457-462. doi:10. 1123/ijspp.2012-0347. PMID:23475154.

Beaven, C.M., Maulder, P., Pooley, A., Kilduff, L., and Cook, C. 2013. Effects of caffeine and carbohydrate mouth rinses on repeated sprint performance. Appl. Physiol. Nutr. Metab. 38(6): 633-637. doi:10.1139/apnm-2012-0333. PMID: 23724880
Beelen, M., Berghuis, J., Bonaparte, B., Ballak, S.B., Jeukendrup, A.E., and vanLoon, L.J. 2009. Carbohydrate mouth rinsing in the fed state: lack of enhancement of time-trial performance. Int. J. Sport Nutr. Exerc. Metab. 19(4): 100-409. PMID:19827464.

Borg, G.A. 1982. Psychophysical bases of perceived exertion. Med. Sci. Sports Exerc. 14(5): 377-381. PMID:7154893.

Borg, G., Hassmén, P., and Lagerström, M. 1987. Perceived exertion related to heart rate and blood lactate during arm and leg exercise. Eur. J. Appl. Physiol. 56(6): 679-685. doi:10.1007/BF00424810. PMID:3678222.

Buchheit, M., and Laursen, P.B. 2013. High-intensity interval training, solutions to the programming puzzle. Sports Med. 43(5): 313-338. doi:10.1007/s40279013-0029-x. PMID:23539308.

Carr, A., Dawson, B., Schneiker, K., Goodman, C., and Lay, B. 2008. Effect of caffeine supplementation on repeated sprint running performance. J. Sports Med. Phys. Fitness, 48(4): 472-478. PMID:18997650.

Carter, J.M., Jeukendrup, A.E., and Jones, D.A. 2004. The effect of carbohydrate mouth rinse on 1-h cycle time trial performance. Med. Sci. Sports Exerc. 36(12): 2107-2111. PMID:15570147.

Chambers, E.S., Bridge, M.W., and Jones, D.A. 2009. Carbohydrate sensing in the human mouth: effects on exercise performance and brain activity. J. Physiol. 587(8): 1779-1794. doi:10.1113/jphysiol.2008.164285. PMID:19237430.

Chong, E., Guelfi, K.J., and Fournier, P.A. 2011. Effect of a carbohydrate mouth rinse on maximal sprint performance in competitive male cyclists. J. Sci. Med. Sport, 14(2): 162-167. doi:10.1016/j.jsams.2010.08.003. PMID:20932798.

Cohen, J. 1992. A power primer. Psychol. Bull. 112: 155-159. doi:10.1037/00332909.112.1.155. PMID:19565683.

Devenney, S., Collins, K., and Shortall, M. 2016. Effects of various concentrations of carbohydrate mouth rinse on cycling performance in a fed state. Eur. J. Sport Sci. 16(8): 1073-1078. doi:10.1080/17461391.2016.1196735. PMID:27339107.

Dorling, J.L., and Earnest, C.P. 2013. Effect of carbohydrate mouth rinsing on multiple sprint performance. J. Int. Soc. Sports Nutr. 10(1): 41. doi:10.1186/ 1550-2783-10-41. PMID:24066731.

Fares, E.-J.M., and Kayser, B. 2011. Carbohydrate mouth rinse effects on exercise capacity in pre- and postprandial states. J. Nutr. Metab. 2011: 385962. doi:10. 1155/2011/385962. PMID:22013515.

Frank, G.K.W., Oberndorfer, T.A., Simmons, A.N., Paulus, M.P., Fudge, J.L., Yang, T.T., and Kaye, W.H. 2008. Sucrose activates human taste pathways differently from artificial sweetener. NeuroImage, 39(4): 1559-1569. doi:10. 1016/j.neuroimage.2007.10.061. PMID:18096409.

Goldstein, E.R., Ziegenfuss, T., Kalman, D., Kreider, R., Campbell, B., Wilborn, C., Taylor, L., et al. 2010. International society of sports nutrition position stand: caffeine and performance. J. Int. Soc. Sports Nutr. 7(1): 5. doi:10.1186/15502783-7-5. PMID:20205813.

Graham, T.E., and Spriet, L.L. 1995. Metabolic, catecholamine, and exercise performance responses to varying doses of caffeine ingestion. J. Appl. Physiol. 78: 867-874. doi:10.1152/jappl.1995.78.3.867. PMID:7775331.

Hopkins, W.G., Marshall, S.W., Batterham, A.M., and Hanin, J. 2009. Progressive statistics for studies in sports medicine and exercise science. Med. Sci. Sports Exerc. 41(1): 3-12. doi:10.1249/MSS.0b013e31818cb278. PMID:19092709.

Kalmar, J.M. 2005. The influence of caffeine on voluntary muscle activation. Med. Sci. Sports Exerc. 37(12): 2113-2119. doi:10.1249/01.mss.0000178219.18086.9e. PMID:16331138.

Kasper, A.M., Cocking, S., Cockayne, M., Barnard, M., Tench, J., Parker, L., et al. 2016. Carbohydrate mouth rinse and caffeine improves high-intensity interval running capacity when carbohydrate restricted. Eur. J. Sport Sci. 16(5): 560-568. doi:10.1080/17461391.2015.1041063. PMID:26035740.

Kizzi, J., Sum, A., Houston, F.E., and Hayes, L.D. 2016. Influence of a caffeine mouth rinse on sprint cycling following glycogen depletion. Eur. J. Sport Sci. 16(8): 1087-1094. doi:10.1080/17461391.2016.1165739. PMID:27686403. 
Lakens, D. 2013. Calculating and reporting effect sizes to facilitate cumulative science: a practical primer for t-tests and ANOVAs. Front. Psychol. 4: 863. doi:10.3389/fpsyg.2013.00863. PMID:24324449.

Lane, S.C., Bird, S.R., Burke, L.M., and Hawley, J.A. 2013. Effect of a carbohydrate mouth rinse on simulated cycling time-trial performance commenced in a fed or fasted state. Appl. Physiol. Nutr. Metab. 38(2): 134-139. doi:10.1139/ apnm-2012-0300. PMID:23438223.

Lee, C.L., Lin, J.C., and Cheng, C.F. 2011. Effect of caffeine ingestion after creatine supplementation on intermittent high-intensity sprint performance. Eur. J. Appl. Phys. 111(8): 1669-1677. doi:10.1007/s00421-010-1792-0.

Lesniak, A.Y., Davis, S.E., Moir, G.L., and Sauers, E.J. 2016. The effects of carbohydrate, caffeine, and combined rinses on cycling performance. J. Sport Hum. Perform. 4(1).

Mohr, M., Nielsen, J.J., and Bangsbo, J. 2011. Caffeine intake improves intense intermittent exercise performance and reduces muscle interstitial potassium accumulation. J. Appl. Physiol. 111(5): 1372-1379. doi:10.1152/japplphysiol.01028. 2010. PMID:21836046.

Pottier, A., Bouckaert, J., Gilis, W., Roels, T., and Derave, W. 2010. Mouth rinse but not ingestion of a carbohydrate solution improves 1-h cycle time trial performance. Scand. J. Med. Sci. Sports, 20(1): 105-111. doi:10.1111/j.1600-0838. 2008.00868.x. PMID:19000099.

Rollo, I., and Williams, C. 2011. Effect of mouth-rinsing carbohydrate solutions on endurance performance. Sports Med. 41(6): 449-461. doi:10.2165/11588730000000000-00000. PMID:21615187.

Rollo, I., Williams, C., Gant, N., and Nute, M. 2008. The influence of carbohydrate mouth rinse on self-selected speeds during a 30-min treadmill run. Int. J. Sport Nutr. Exerc. Metab. 18(6): 585-600. doi:10.1123/ijsnem.18.6.585. PMID: 19164829.

Rollo, I., Cole, M., Miller, R., and Williams, C. 2010. Influence of mouth rinsing a carbohydrate solution on 1-h running performance. Med. Sci. Sports Exerc. 42(4): 798-804. doi:10.1249/MSS.0b013e3181bac6e4. PMID:19952850.

Turner, C.E., Byblow, W.D., Stinear, C.M., and Gant, N. 2014. Carbohydrate in the mouth enhances activation of brain circuitry involved in motor performance and sensory perception. Appetite, 80: 212-219. doi:10.1016/j.appet.2014.05. 020 .

Whitham, M., and McKinney, J. 2007. Effect of a carbohydrate mouthwash on running time-trial performance. J. Sports Sci. 25(12): 1385-1392. doi:10.1080/ 02640410601113676. PMID:17786691. 